\title{
The Stethoscope, Respiratory Examination and Lung Ultrasound in the COVID-19 Era
}

Dr Avinash Aujayeb, corresponding author

Northumbria HealthCare NHS Foundation Trust

Care of Tracy Groom, Northumbria Way, Cramlington, Northumberland, NE23 6NZ

Tel: 07703343329

Email: avinash.aujayeb@nhct.nhs.uk

Authorship statement: AA wrote the whole manuscript

Disclosure statement: Conflicts DO NOT exist.

\section{Abstract:}

The current Covid-19 pandemic has hugely disrupted the delivery of routine and established medical care. Patients can develop a wide range of clinical signs and symptoms from a cough and fever to severe respiratory failure. There is an ongoing argument on a concise investigative pathway to ensure the safety of all healthcare workers. The stethoscope can help with any clinical respiratory assessment but the risk of cross infection is high. Computer tomography should not be routinely performed. There is a potential place for lung ultrasound but outcomes are not yet determined.

Keywords: respiratory failure; stethoscope; ultrasound; Covid-19 


\section{Introduction}

The covid-19 era poses significant and unprecedented challenges for re-organisation of care, redistribution of resources and rationing of services [1,2]. A review of patients' clinical characteristics showed a high prevalence of respiratory symptoms and respiratory failure which require full assessment [3]. There is a view that the stethoscope has limited usefulness in that assessment [4]. The respiratory examination is taught in medical schools all over the world and forms an integral part of some postgraduate examinations. Further investigations usually are a chest radiograph (CXR) and if there is still diagnostic doubt, or need to narrow down the differential diagnoses, a computer tomogram (CT) can be performed $[5,6]$.

\section{The stethoscope in respiratory failure}

In 1816, a French physician, René Laennec, invented the stethoscope and was also the first to describe the terms rales, rhonchi, crepitance, and egophony which are associated with specific respiratory conditions [7]. Over time, acquiring and wearing a stethoscope has become a rite of passage for many doctors and has formed an integral part of the diagnostic pathway [8]. The respiratory examination comprises of four basic tenets: inspection, palpation, percussion and auscultation. Auscultation requires the stethoscope to be applied to the front and back of chest, as well as under the axillae. However, the signs are neither specific nor sensitive. The main sign in respiratory failure syndromes is crackles [9]. Crackles on auscultation have a sensitivity of $19-67 \%$ and a specificity of $36-96 \%$, with a positive likelihood ration of 2.3 and a negative likelihood ration of 0.8 [9]. Thus, their use in ruling pneumonia in or out is limited as their presence or absence only slightly changes the initial diagnosis (poor inter observer reliability, 72\% agreement, kappa value 0.41) [10]. Lovrenski et al replicated similar findings in children with suspected pneumonia [11]. Hence, given the high incidence of interstitial changes and respiratory failure in Covid-19 and the risk of cross infection with taking a stethoscope from a patient to the next, the stethoscope should be disregarded $[3,4]$.

\section{CXRs and CTs in the Covid-19 era}

The current worldwide pandemic of Covid-19 (SARS-CoV-2)) started in China and has now spread around the world. At the time of writing, latest figures show just over 550, 000 confirmed cases and 26,000 deaths related to it [12]. Measures to curb the spread of the disease have included social distancing, closure of schools and non-essential work, closure of borders, cessation of international travel and lockdown of entire cities. This has been well documented in the literature and mainstream media [13]. However, it is primordial that the healthcare workers assessing suspected or confirmed cases takes clear steps to avoid the spread of Covid-19 to other patients with the possible creation 
ofnew outbreaks. Patients have a high incidence of respiratory symptoms that require assessment [3]. In the early days of the outbreak, some Chinese centres ran out of polymerase chain reaction (PCR) testing kits, which only have an approximate sensitivity of $60-70 \%$ and chest computed tomography (CT) scans were used as a diagnostic tool. In a review of 113 deceased patients from China, $100 \%$ of the deceased patients and $94 \%$ of the recovered patients had bilateral changes on chest radiography [3]. Typical findings on CT images are bilateral ground glass opacities and subsegmental areas of consolidation. The British Thoracic Society of Imaging has produced some guidance to minimise risk to staff and reduce transferring patient to and from the radiology department and reduce CT scanning as well as deep cleaning in between scans. The decision tool suggests that there is no role for CT imaging in the diagnosis of COVID-19 unless the patient is seriously ill or if PCR is unavailable [14].

\section{Does lung ultrasound have a role?}

Lung ultrasonography in acute respiratory failure has been described for over a decade. A 2008 single centre study of bedside lung ultrasound examination protocol showed an accuracy of $90.5 \%$ in diagnosing the cause of acute respiratory failure in critically ill patients. The methods were standardised and reproducible [15]. This has been since incorporated in consensus guidelines [16]. As such, it has been debated as to whether lung ultrasound can have a role to play in the diagnostic pathway for Covid-19. The vast majority of point of care ultrasound machines are small, portable and can be easily cleaned in between patients. Huang et al performed lung ultrasound on 19 non-critical Covid-19 patients and found them to have specific characteristics (large number of B lines, subpleural pulmonary consolidation and poor blood flow) which were mainly visible in the posterior and inferior areas [17]. Ultrasound missed lesions that were completely intrapulmonary and apical and some of the analysis is only in abstract form. Care must be applied to the analysis of this as this is a pre-print, and not peer-reviewed. Peng et al found similar findings using a similar 12 zone method but the article lacks details about the clinical characteristics of the patients [18]. Poggiali et al found strong correlation between similar ultrasound findings and strong correlation between ultrasound and CT scan findings [19].

Soldati et al propose a standard process for the use of lung ultrasound for COVID-19 patients but so far there have been no studies looking at patient outcomes [20]. Newly designed guidance from the Association of Chartered Physiotherapists in Respiratory Care are laudable and eventual data gathered from the application of lung ultrasound will be crucial [21].

A quick survey of 40 patients with confirmed Covid-19 was performed locally. All of them were on the respiratory wards and 12 were receiving continuous positive airway pressure (CPAP) ventilation. No 
patients had a respiratory examination when they first presented, all had abnormal CXRs and none had had a CT scan. None had had a lung ultrasound either. The question is whether these patients had a lung ultrasound initially, would it have changed their management? That is unlikely, as they were all getting admitted into a side room and being swabbed. Swabs results are available locally within a few hours. A quick survey of 12 patients whose first swabs were negative for Covid-19 was also done. All were treated as being high suspicion for Covid-19 and were also in side rooms. All had abnormal CXRs and none had CTs. 6 were receiving CPAP. The only difference in management between the two groups was that the confirmed Covid-19 patients were all enrolled in the Recovery trial [22]. However, the inclusion criteria into that particular trial has now changed, and patients not confirmed to have, but with high suspicion of Covid-19 are eligible too.

\section{Conclusions}

From the current evidence and practice, stethoscopes and CT scans have minimal roles in Covid-19 and only if there is significant diagnostic uncertainty. Whilst lung ultrasound has been shown to demonstrate specific characteristics in Covid-19, there is no current evidence for lung ultrasound changing management and affecting patient outcomes. The vast majority of physicians are not trained at lung ultrasound and that will prove a significant barrier for widespread use. Other foreseeable barriers are the availability of ultrasound machines, and the manpower and time required to clean machines after use on a patient. Perhaps lung ultrasound may have a role to play in triage of suspected Covid-19 patients in resource limited settings but again this has not been studied or reported. 


\section{References}

1. Extance Andy. Covid-19 and long term conditions: what if you have cancer, diabetes, or chronic kidney disease? BMJ 2020; 368 :m1174

2. Willan John, King Andrew John, Jeffery Katie, Bienz Nicola. Challenges for NHS hospitals during covid-19 epidemic BMJ 2020; 368 :m1117

3. Chen T, Wu D, Chen H, Yan W, Yang Di, Chen G, Ma K et al. Clinical characteristics of 113 deceased patients with coronavirus disease 2019: retrospective, study BMJ 2020; 368 :m1091

4. Buensonso D, Pata D, Chiaretti A COVID-19 outbreak: less stethoscope, more ultrasound Lancet Respir Med 2020 Published Online March 20, 2020 https://doi.org/10.1016/ S2213$2600(20) 30120-X$

5. https://www.medistudents.com/en/learning/osce-skills/respiratory/respiratoryexamination/ (accessed 28.3.2020)

6. https://www.mrcpuk.org/mrcpuk-examinations/paces/paces-examination-format (accessed 28.3.2020)

7. Scherer, John R. (2007). "Before cardiac MRI: Rene Laennec (1781-1826) and the invention of the stethoscope". Cardiology Journal. 14 (5): 518-519.

8. Copetti R Is lung ultrasound the stethoscope of the new millennium? Definitely yes! Acta Med Acad. 2016 May;45(1):80-1. doi: 10.5644/ama2006-124.162.

9. Simel DL, Rennie D, Keitz SA. The rational clinical examination: evidence-based clinical diagnosis. McGraw-Hill, 2009.

10. Metlay JP, Kapoor WN, Fine MJ. Does this patient have community-acquired pneumonia? Diagnosing pneumonia by history and physical examination. JAMA 1997;278:1440- 
11. Lovrenski J, Slobodanka Petrović S, Balj-Barbir S et al Stethoscope vs. ultrasound probe which is more reliable in children with suspected pneumonia? Acta Med Acad.

$2016 ; 45(1): 39-50$

12. https://www.who.int/emergencies/diseases/novel-coronavirus-2019/situation-reports (accessed 29.3.2020)

13. Anderson R, Heesterbeek H, Klinkenberg D, Hollingswroth TD How will country-based mitigation measures influence the course of the COVID-19 epidemic? The Lancet Volume 395, Issue 10228, p931-934, March 21, 2020

14. https://www.bsti.org.uk/media/resources/files/BSTI_COVID19_Radiology_Guidance_version_2_16.0 3.20.pdf (accessed 29.3.2020)

15. Silva S, Biendel C, Ruiz J, Olivier M, Bataille B, Geeraerts T, Mari A, et al . Usefulness of cardiothoracic chest ultrasound in the management of acute respiratory failure in critical care practice. Chest 2013;144:859-65. 16. Lichtenstein DA, Malbrain MLNG. Lung ultrasound in the critically ill (LUCI):

a translational discipline. Anaesthesiol Intensive Ther 2017; 49: 430-36.

17. https://www.researchsquare.com/article/rs-14928/v1 (accessed 29.3.2020)

18. Peng, Q., Wang, X. \& Zhang, L. Findings of lung ultrasonography of novel corona virus pneumonia during the 2019-2020 epidemic. Intensive Care Med 2020 Mar 12. doi: 10.1007/s00134-020-05996-6. [Epub ahead of print] 19. Poggiali E, Dacrema A, Bastoni D, Tinelli V, Demichele E, Mateo Ramos P, Marcianò T et al Can Lung US Help Critical Care Clinicians in the Early Diagnosis of Novel Coronavirus (COVID-19) Pneumonia? Radiology 2020 Mar 13:200847 20. https://onlinelibrary.wiley.com/doi/epdf/10.1002/jum.15285 (accessed 5.4.2020) 
21.https://www.acprc.org.uk/Data/Resource_Downloads/PhysioLUSCOVID19.pdf?date=07/04/2020 \%2003:35:15 (accessed 10.4.2020)

22. https://www.recoverytrial.net/ (10.4.2020) 